\title{
Manipulation of Nuclear Spins in Interfaces of Diluted Magnetic Semiconductors *
}

\author{
M. Ogura ${ }^{\dagger}$ and H. Akai \\ Department of Physics, Graduate School of Science, Osaka University, \\ 1-1 Machikaneyama, Toyonaka, Osaka 560-0043, Japan \\ (Received 30 October 2007; Accepted 12 December 2007; Published 16 January 2008)
}

\begin{abstract}
A way of nuclear spin manipulation using an interface of diluted magnetic semiconductors (DMSs) is proposed. The hyperfine field at an impurity site in the interface of a DMS and a base substance can be controlled by applying the external electric field. It is because that the electric field changes the spin density of carriers in the $s$ state at the impurity site. On the basis of first-principles electronic structure calculation, we investigated the electric-field dependence of the hyperfine field at the impurity site. We show that the hyperfine field is dramatically changed by an external electric field when no clear magnetic impurity band is formed in the band gap.

[DOI: $10.1380 /$ ejssnt.2008.7]
\end{abstract}

Keywords: Density functional calculations; Nuclear magnetic resonance; diluted magnetic semiconductor; hyperfine field

\section{INTRODUCTION}

Numerous attempts have been made towards realization of quantum computing. The spin degree of freedom is one of candidates for quantum bits. Especially nuclear spins are suitable for this purpose because their relaxation time is much longer than electron spins. In order to realize the control on the nuclear spins, nuclear magnetic resonance (NMR) using electrics fields or electric currents has been investigated by several authors [1-4]. It is clear that NMR using electric field is more practical than that using magnetic fields in semiconductor devices. In those studies, the possibilities of controlling a hyperfine field by changing the electric field of targeted nuclei were pointed out.

In the present study, we propose a new method of the nuclear spin manipulation. This method uses an interface of diluted magnetic semiconductors (DMSs) $[5,6]$. Here, we consider a hetero-structure of DMSs and base substances with impurities in the vicinity of the interface. We focus on the hyperfine field at the impurity site. We examine the electronic structure of the interfaces of DMSs under an electric field on the basis of the first-principles electronic structure calculation and show that the hyperfine field can be controlled by the electric field.

\section{MECHANISM}

Our system uses the hetero-structure of DMSs, say, (In,Mn)As, and base substances, for example, AlSb. In the ferromagnetic DMSs, the local magnetic moments of the magnetic ions couple to each other through the double exchange between the $d$ states of neighboring magnetic ions or the $p$ - $d$ hybridization between the magnetic $d$ states and the $p$ states of surrounding anions. In this situation, carrier holes exist as if they are mediating the magnetic coupling.

Consider an impurity in DMS located near the inter-

* This paper was presented at Handai Nanoscience and Nanotechnology International Symposium (Handai Nano 2007), Osaka University, Japan, 26-28 September, 2007.

${ }^{\dagger}$ Corresponding author: ogura@phys.sci.osaka-u.ac.jp face of the DMS and base substance. The impurity should be chosen so that the $s$ states of the impurity are energetically near the Fermi level. The impurity levels are spin split due to the strong hybridization with the $p$ and $d$ states of DMS. The schematic view of the electronic structure in this situation is shown in Fig. 1. In the figure, the impurity $s$ states are fully occupied by holes and show no significant $s$ spin polarization. This implies that the hyperfine field seen by the impurity nucleus is nearly zero. However, if we could slightly decrease the hole density in the impurity $s$ state, it would immediately cause a decrease in the number of spin-up holes, thus producing a negative spin polarization and a negative hyperfine field. In this manner, one can control the hyperfine field by adjusting the hole concentration in the vicinity of the interface of the system.

\section{CALCULATION}

Calculation was performed using the Kohringa-KohnRostoker (KKR) Green's function method combined with the coherent potential approximation (CPA) and the local spin density approximation (LSDA) of the density functional theory. In the framework of CPA, the host atoms

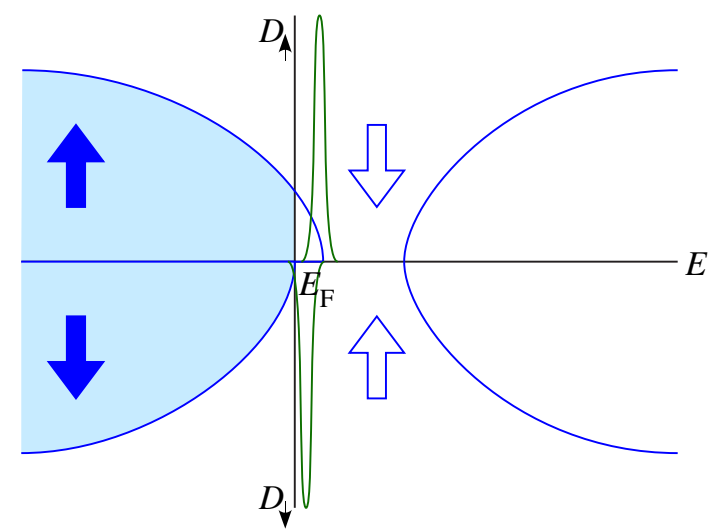

FIG. 1: Schematic views of the electronic structure of DMS with an impurity. The blue lines show the total density of states and the shaded areas indicate occupied states. In this situation, spin-down carrier holes exist. The green lines show the $s$ state of the impurity. The filled and open arrows denote spins for electrons and holes, respectively. 


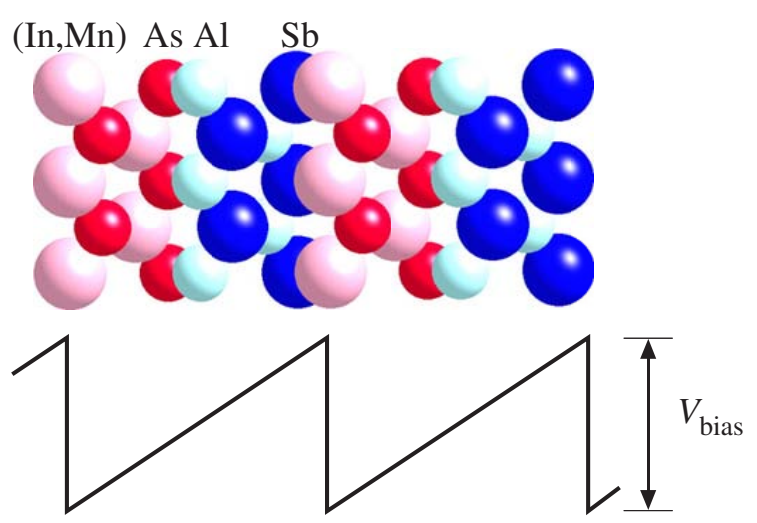

FIG. 2: Superlattice of (InAs) $)_{2}(\mathrm{AlSb})_{2}$ and a periodic potential used in this work.

are randomly substituted by impurities and the configuration average with respect to the random arrangements of the impurities is taken [7]. From the obtained electronic structures, hyperfine fields are calculated [8].

DMSs are classified into two types: One is the DMS which has clear impurity bands in the band gap. In such a DMS, ferromagnetism is stabilized in the impurity bands through the double-exchange interaction. Most II-VI type DMSs such as (ZnCr)Te and (ZnMn)Se are included in this type. On the other hand, most of III-V type DMSs such as (InMn)As and (GaMn)As do not show such clear impurity bands in the band gap. In this case, the ferromagnetism is induced in large extent by the $p-d$ hybridization [7]. In the present study, we examined (InMn)As and $(\mathrm{ZnCr}) \mathrm{Te}$ in order to compare these different types of DMSs.

To simulate the hetero-structure of these DMSs, we used superlattices of a DMS and a semiconductor with a zinc-blende structure. As an example, the super lattice of (InMn)As and AlSb is shown in Fig. 2. Impurities are supposed to substitute for In or Zn. A periodic sawtooth-like potential simulates an applied electric field as is also shown in Fig. 2. This gives an external constant field through the unit cell. The jump in the potential causes an infinite field. This however is not a problem since the effect is to reset the boundary condition, simulating the electromotive force of an electric generator or a battery.

\section{RESULTS AND DISCUSSION}

Figure 3 shows the density of states (DOS) of the hetero-structure of $\left(\operatorname{In}_{0.95} \mathrm{Mn}_{0.05}\right) \mathrm{As}$ and AlSb. As impurities, we considered an antisite As substituting for In. We can see that the Mn $d$-states (spin-up) are located at the low energy side because of the $p-d$ hybridization. The main contribution to the hyperfine fields is the Fermi contact interaction, where the polarization of $s$ electrons play a role. Therefore the $s$-state at the impurity site is important. The $s$ states of an impurity, namely the antisite As, are formed in the band gap and spin split due to the strong hybridization with the As $p$ - and $\mathrm{Mn} d$-states. Without the bias voltage, the impurity states are located above the Fermi level and are not occupied. This means that the hyperfine field seen by the As nucleus is nearly zero. However, when bias voltage is applied, the state gradually
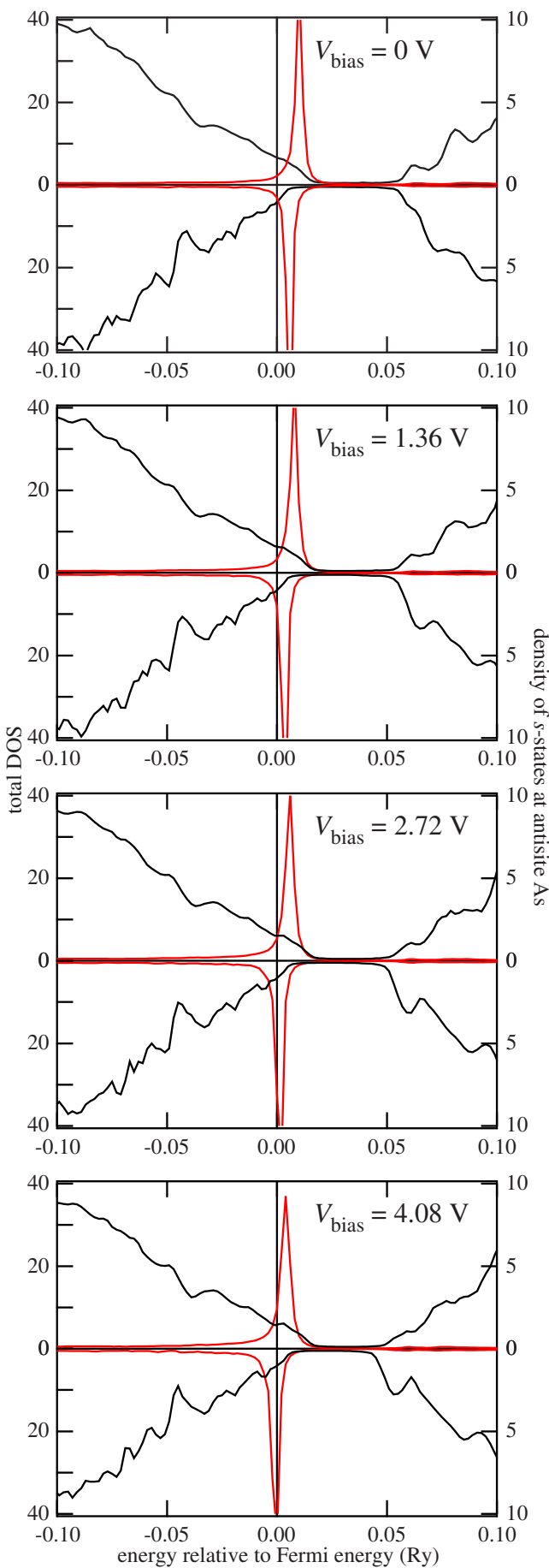

FIG. 3: DOS for $\left(\operatorname{In}_{0.95} \mathrm{Mn}_{0.05} \mathrm{As}_{0}\right) \mathrm{As} / \mathrm{AlSb}$. The black lines show the total DOS (left axis). The red lines show the local $s$ state of the antisite As (right axis).

moves down to the lower energy side, and finally it comes below the Fermi level. Since even the bound states have a finite width (virtual bound states) due to the coupling with other antisite As, a slight shift of the level across the Fermi level does not mean any sudden jump in the occupation number. In fact, the change in the occupation is tiny in this case. At $V_{\text {bias }} \sim 4 \mathrm{~V}$, the spin-down electron $s$ state is almost fully occupied, whereas the spin-up electron state is not occupied.

The change in the $s$ electron polarization causes a large change in the hyperfine field seen by the As nucleus. Figure 4 shows the bias voltage dependence of the hyperfine 


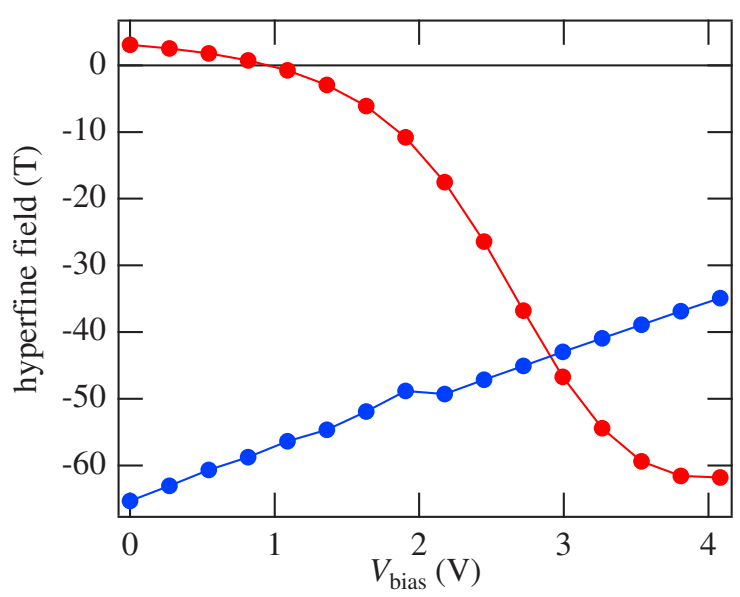

FIG. 4: Bias voltage dependence of the hyperfine field at the antisite $\mathrm{As}$ in $\left(\mathrm{In}_{0.95} \mathrm{Mn}_{0.05} \mathrm{As}_{0}\right) \mathrm{As} / \mathrm{AlSb}$ (red) and $\mathrm{P}$ in $\left(\mathrm{Zn}_{0.95} \mathrm{Cr}_{0.05} \mathrm{P}_{0}\right) \mathrm{Te} / \mathrm{ZnTe}$ (blue).

field at the antisite As. The hyperfine field changes considerably by the applied electric field and it has a large negative value at a certain bias voltage. This implies that we can control the hyperfine field at the impurity site near the interface via electric fields.

The situation is quite different in the case of $(\mathrm{ZnCr}) \mathrm{Te}$. Figure 5 shows the DOS of the hetero-structure of $\left(\mathrm{Zn}_{0.95} \mathrm{Cr}_{0.05}\right) \mathrm{Te}$ and $\mathrm{ZnTe}$. As impurities, we considered $\mathrm{P}$ substituting for Zn. For the DOS of spin-up state, a clear impurity band of $\mathrm{Cr} d$ states appears in the band gap. Without a bias voltage, the spin-down $s$ state at $\mathrm{P}$ is fully occupied, whereas the spin-up state has holes. Hence the $\mathrm{P}$ nucleus feels a finite hyperfine field. However, the spin-up $s$ states of $\mathrm{P}$ is broader than those of antisite As in (InMn)As because of a strong hybridization with $\mathrm{Cr} d$ states. When bias voltage is applied, the $\mathrm{P} s$ state stays at almost the same energy position whereas the total DOS gradually moves. This means that the hyperfine field seen by the $\mathrm{P}$ nucleus does not change much.

Figure 4 also shows the bias voltage dependence of the hyperfine field at $\mathrm{P}$ in $\left(\mathrm{Zn}_{0.95} \mathrm{Cr}_{0.05}\right) \mathrm{Te} / \mathrm{ZnTe}$ heterostructure. Though the hyperfine field has a negative large value, the change caused by the applied electric field is not as drastic as that for the antisite As in $\left(\mathrm{In}_{0.95} \mathrm{Mn}_{0.05}\right) \mathrm{As} / \mathrm{AlSb}$. For this reason, it is rather difficult to control the hyperfine field via the electric field in such systems.

\section{CONCLUSION}

We have proposed a new nuclear spin manipulation technique, which uses an interface of DMSs and is driven by electric fields. Our first-principles calculation predicts significant changes in the hyperfine fields induced by applying a bias voltage in the case of the III-V DMSs. The behaviors of the hyperfine field under the bias voltage can be controlled by choosing the base substances and the concentration of magnetic impurities. They can be also controlled by carrier doping. In contrast, such manipulation seems rather difficult in the case of II-VI DMSs. If the control of the hyperfine field is possible for each impurity or each cluster of impurities, they can be used as
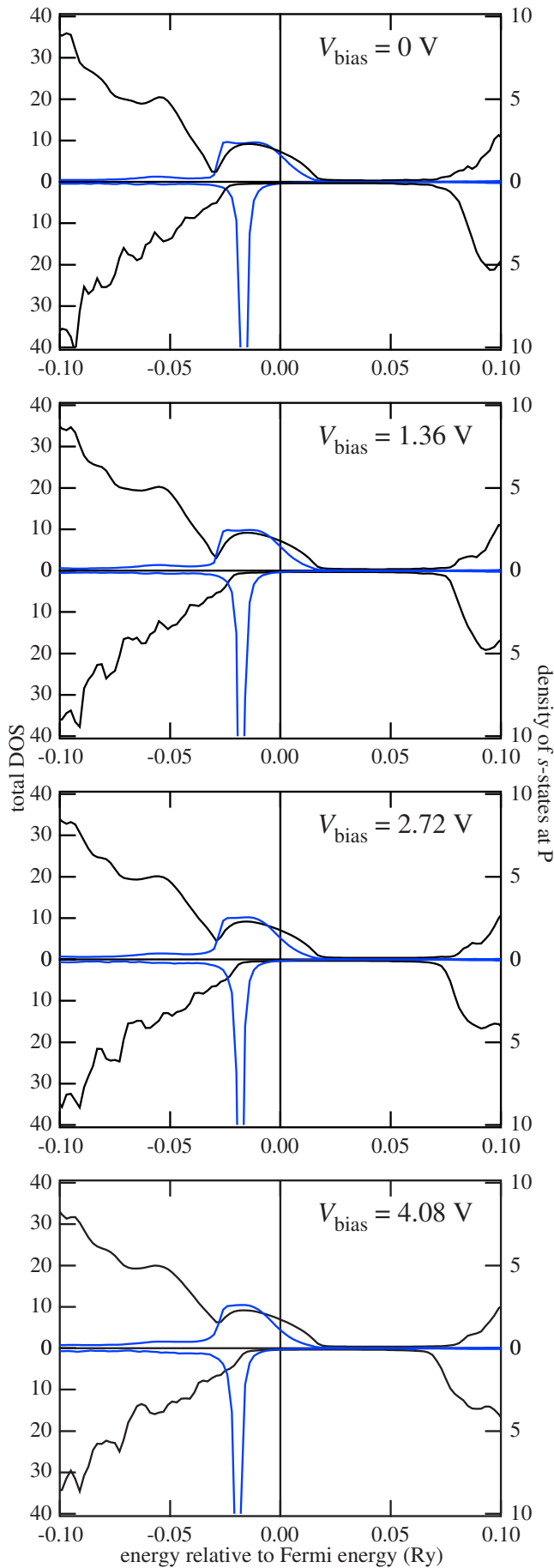

FIG. 5: DOS for $\left(\mathrm{Zn}_{0.95} \mathrm{Cr}_{0.05} \mathrm{P}_{0}\right) \mathrm{Te} / \mathrm{ZnTe}$. The black lines show the total DOS (left axis). The blue lines show the local $s$ state of $\mathrm{P}$ (right axis).

quantum bits. The study for such applications is now in progress.

\section{Acknowledgments}

This work is partly supported by Special Coordination Funds for the Promotion of Science and Technology, Leading Research "Nanospintronics Design and Realization", by MEXT KAKENHI, No.16031210, 17064008, by JSPS KAKENHI No. 17740194, and by the Next Generation 
Super Computing Project, Nanoscience Program, MEXT.

[1] B. E. Kane, Nature 393, 133 (1998).

[2] T. Machida, T. Yamazaki, K. Ikushima, and S. Komiyama, Appl. Phys. Lett. 82, 409 (2003).

[3] G. Yusa, K. Muraki, K. Takashina, K. Hashimoto, and Y. Hirayama, Nature 434, 1001 (2005).

[4] H. Takahashi, M. Kawamura, S. Masubuchi, K. Hamaya, and T. Machida, Appl. Phys. Lett. 91, 092120 (2007).

[5] H. Munekata, H. Ohno, S. von Molnar, A. Segmuller, L. L.
Chang, and L. Esaki, Phys. Rev. Lett. 63, 1849 (1989).

[6] H. Ohno, A. Shen, F. Matsukura, A. Oiwa, A. Endo, and Y. Iye, Appl. Phys. Lett. 69, 363 (1996).

[7] H. Akai, Phys. Rev. Lett. 81, 3002 (1998).

[8] H. Akai, M. Akai, S. Blügel, et al., Prog. Theor. Phys. Suppl. 101, 11 (1990). 